\title{
A case of prenatal diagnosis of 16q24.3 microdeletion KBG syndrome and review of the literature
}

\author{
tian qin deng ${ }^{1}$, qingzhi liu ${ }^{1}$, jiansheng $x^{2}{ }^{2}$, xuemei li $^{2}$, and bing yao ${ }^{1}$ \\ ${ }^{1}$ Southern Medical University Clinical Medical College \\ ${ }^{2}$ Shenzhen Maternity and Childcare Hospital
}

July 20, 2021

\begin{abstract}
Diagnosis of KBGS due to a high risk identified by early Down's syndrome screening has not been reported in previous studies, and the prominent KBGS phenotype and absence of specificity on early ultrasound examination also pose a challenge for genetic diagnosis.
\end{abstract}

A case of prenatal diagnosis of 16q24.3 microdeletion KBG syndrome and review of the literature

Tianqin Deng ${ }^{1,2}$ Qingzhi Liu ${ }^{2}$ Jiansheng Xie ${ }^{2}$ Xuemei $\mathrm{Li}^{2}$ Bing Yao ${ }^{1 *}$

1 Reproductive Medical Center,Nanjing School of Clinical Medicine,Southern Medical University(General Hospital of Eastern Military Region),305 Zhongshan Dong Road, Xuanwu District, Nanjing, Jiangsu Province, China

2 Reproductive Medical Center,Affiliated Shenzhen Maternity \& Child Healthcare Hospital, Southern Medical University (Shenzhen Maternity \& Child Healthcare Hospital),3012 Fuqiang Road, Futian District, Shenzhen, Guangdong Province, China

*Corresponding author:BingYao,Email:yaobing@nju.edu.cn,

Address:305 Zhongshan Dong Road, Xuanwu District, Nanjing, Jiangsu Province, 210002,China.

\section{Key Clinical Message}

KBG syndrome is a rare autosomal dominant genetic disease mainly caused by pathogenic variants of ankyrin repeat domain-containing protein 11 (ANKRD11) or deletions involving ANKRD11.we report a case of KBGS in a fetus that was prenatally diagnosed due to the high risk identified by early Down's syndrome screening and the finding of chromosome 16q24.3 deletion by chromosomal microarray analysis (CMA).

Key words :KBG syndrome;ANKRD11;chromosome microarray analysis

\section{INTRODUCTION}

KBG syndrome (KBGS) is a rare autosomal dominant genetic disorder characterized by extensive growth retardation, craniofacial anomalies, oversized maxillary central incisors and skeletal anomalies, as well as genetic alterations such as ANKRD11 gene mutation and 16q24.3 microdeletion (Gnazzo,2020), and prenatal diagnosis of KBGS has rarely been reported in China. In this study, we prenatally diagnosed KBGS in a foetus and analysed its possible mechanism to provide a basis for clinical diagnosis, genetic counselling and prenatal diagnosis. 


\section{methods}

\section{Subject}

A pregnant woman, 31 years old, in her second pregnancy and first delivery received early Down's syndrome serological screening at 12 weeks of gestation. The results suggested that the chance of trisomy 21 was 1:89, which is high risk. Prenatal assessment was performed to further clarify the risk. After informed consent was obtained from the pregnant woman, amniocentesis was employed for amniotic fluid karyotyping and chromosome microarray analysis. Termination of pregnancy was chosen after genetic counselling and multidisciplinary consultation, but an autopsy was refused. The study was approved by the hospital ethics committee, and the pregnant woman and her relatives signed an informed consent form.

\section{Methods}

Amniotic fluid karyotype analysis Foetal amniotic fluid $(30 \mathrm{~mL})$ was extracted via ultrasound-guided transabdominal amniotic cavity puncture at mid-pregnancy, of which $10 \mathrm{~mL}$ was sent for gene chip analysis, and $20 \mathrm{~mL}$ was placed in 2 sterile centrifuge tubes in a 37 and $5 \% \mathrm{CO} 2$ incubator for cell culture. Cells were harvested by trypsin digestion and imaged and subjected to G-banding analysis, in which more than 20 mid-phase divisions were counted, and karyotype analysis.

CMA assay Genomic DNA was extracted using a QIAamp DNA Mini Kit from Qiagen, Germany. The CMA assay strictly followed the standard operating procedure of the Affymetrix Cytoscan $750 \mathrm{~K}$ chip. Genomic DNA was digested into short fragments with enzymes, ligated with connectors and then amplified by PCR. After that, purification was performed by the magnetic bead method. The purified product was fragmented into 25-125 bp fragments and labelled with biotin. The products were denatured by mixing with hybridization solution, hybridized, washed, stained, and scanned by GeneChip Scanner 3000Dx v.2 with Autoloader, and the data were analysed by ChAS3.0 and related bioinformatics software.

\section{Results}

Amniotic fluid karyotype analysis revealed the following karyotype: 46,XN.

Chromosome microarray analysis revealed the following results: arr[GRCh37] 16q24.3(89261399_89421499)x1, chromosome 16q24.3 deletion (approximately $160 \mathrm{~kb}$; containing a total of 6 genes, 2 of which are OMIM genes: CDH15, SLC22A31, ZNF778, ANKRD11, LOC105371414, and LOC100287036) (Figure 1). The 16q24.3 deletion region was located within the KBGS-related region and contained a partial deletion of the key KBGS gene ANKRD11 (exons 4-14).

Peripheral blood high-throughput sequencing High-throughput sequencing of the pregnant woman's peripheral blood revealed the following results: seq[GRCh37](1-22)x2,(X)x2.

High-throughput sequencing of the patient's husband's peripheral blood revealed the following results: seq[GRCh37](1-22)x2,(XY)x1.

\section{Discussion}

KBGS (OMIM 148050) was first reported in 1975 (Herrmann,1975). The ANKRD11 gene on the long arm of chromosome 16 is the causative gene of KBGS, which features ANKRD11 mutation and the 16q24.3 microdeletion (Gnazzo,2020). Karen Low et al (Low,2016)summarized the clinical and genetic features of 32 cases of KBGS in foetuses, and reported that the foetuses had few identifiable imaging features during the pregnancy. Most of the 20-week anomaly scans were normal apart from single instances of a single umbilical artery, atrioventricular septal defect (AVSD), placenta praevia, and decreased foetal movement. All babies reached a gestation age of at least 36 weeks, and the vast majority were delivered at term. In 2017, Victoria Hodgetts Morton et al (Hodgetts ,2017) reported a case of KBGS in a foetus with a 16q24.3 microdeletion that was prenatally diagnosed due to enhanced intestinal echogenicity, but the autopsy revealed a more severe phenotype: triangular face pattern, hypoplastic ears, cryptorchidism, and pulmonary dysplasia. Novara et al (Novara,,2017)compared the phenotypes of KBGS with ANKRD11 mutation and KBGS with 16q24.3 
microdeletion and found that KGBS with 16q24.3 microdeletion had a more severe phenotype. Goldenberg et al. reported two cases of KBGS with 16q24.3 microdeletion involving ANKRD11 and nearby gene deletions that included clinical manifestations of multiple malformations and learning disabilities (Goldenberg,2016).

In 2012, Japanese scholars reported a clinically confirmed case of KBGS in a patient with a 690-kb deletion on chromosome 16q24.3 (a de novo mutation involving part of ANKRD11) with typical facial appearance, massive maxillary central incisors, hand deformity, delayed bone age and mental retardation, without autistic symptoms (Miyatake,2013) Our reported case also had a de novo mutation with partial deletion of ANKRD11, similar to that reported by the Japanese scholars, and no abnormality was seen on ultrasound at 22 weeks of gestation in our patient, posing a challenge for genetic counselling. The multidisciplinary consultation and literature education informed the patient that (1) the clinical phenotypes of cases with deletions are generally more severe than those of cases with duplications; (2) the larger the deletion or duplicated fragment is, the more severe the manifestation (though the $160 \mathrm{~kb}$ fragment detected in the foetus was a relatively small microdeletion, the region contains the key KBGS pathogenic gene ANKRD11 (deletion of exons 4-10), and partial deletion of exons of this gene presumably leads to severe defects in the function of the encoded protein); (3) de novo mutations are generally highly pathogenic; and (4) postnatal clinical manifestations such as delayed language development, mental retardation, learning disabilities, dwarfism, epilepsy and autism may occur in the foetus, and dwarfism is a common symptom of KBGS for which there is no specific treatment.

\section{CONCLUSION}

The diagnosis of KBGS with microdeletion type 16q24.3 was made in this case due to the high risk identified by early Down's syndrome screening and the finding of chromosome 16q24.3 deletion by chromosomal microarray analysis (CMA). Diagnosis of KBGS due to a high risk identified by early Down's syndrome screening has not been reported in previous studies, and the prominent KBGS phenotype and absence of specificity on early ultrasound examination also pose a challenge for genetic diagnosis.

\section{ETHICS APPROVAL}

This research was approved by the Medical Ethics Committee of Affiliated Shenzhen Maternity\&Child Healthcare Hospital, Southern Medical University (No.LLYJ2021-077-044), and patients signed informed consent forms to participate.

\section{DATA AVAILABILITY STATEMENT}

The data that support the findings of this study are available from the corresponding author upon reasonable request.

\section{Authorship}

Qingzhi Liu ,Jiansheng Xie and Xuemei Li were actively Participated in collection and diagnosis the patient. Tianqin Deng: wrote the manuscript. Bing Yao revised the manuscript.

\section{REFERENCES}

[1]Gnazzo M, Lepri FR, Dentici ML, et al. KBG syndrome: Common and uncommon clinical features based on 31 new patients. Am J Med Genet A . 2020;182(5):1073-1083.

[2]Herrmann J, Pallister PD, Tiddy W, Opitz JM. The KBG syndrome-a syndrome of short stature, characteristic facies, mental retardation, macrodontia and skeletal anomalies. Birth Defects Orig Artic Ser. 1975;11(5):7-18.

[3]Gnazzo M, Lepri FR, Dentici ML, et al. KBG syndrome: Common and uncommon clinical features based on 31 new patients. Am J Med Genet A . 2020;182(5):1073-1083.

[4]Low K, Ashraf T, Canham N, et al. Clinical and genetic aspects of KBG syndrome. Am J Med Genet A . 2016;170(11):2835-2846. 
[5]Hodgetts Morton V, Quinlan-Jones E, Butts N, et al. The first antenatal diagnosis of KBG syndrome: a microdeletion at chromosome 16q24.2q24.3 containing multiple genes including ANKRD11 associated with the disorder. Clin Case Rep . 2017;6(1):189-191.

[6]Novara F, Rinaldi B, Sisodiya SM, et al. Haploinsufficiency for ANKRD11-flanking genes makes the difference between KBG and 16q24.3 microdeletion syndromes: 12 new cases. Eur J Hum Genet . 2017;25(6):694701

[7]Goldenberg A, Riccardi F, Tessier A, et al. Clinical and molecular findings in 39 patients with KBG syndrome caused by deletion or mutation of ANKRD11. Am J Med Genet A . 2016;170(11):2847-2859.

[8]Miyatake S, Murakami A, Okamoto N, et al. A de novo deletion at 16q24.3 involving ANKRD11 in a Japanese patient with KBG syndrome. Am J Med Genet A . 2013;161A(5):1073-1077.

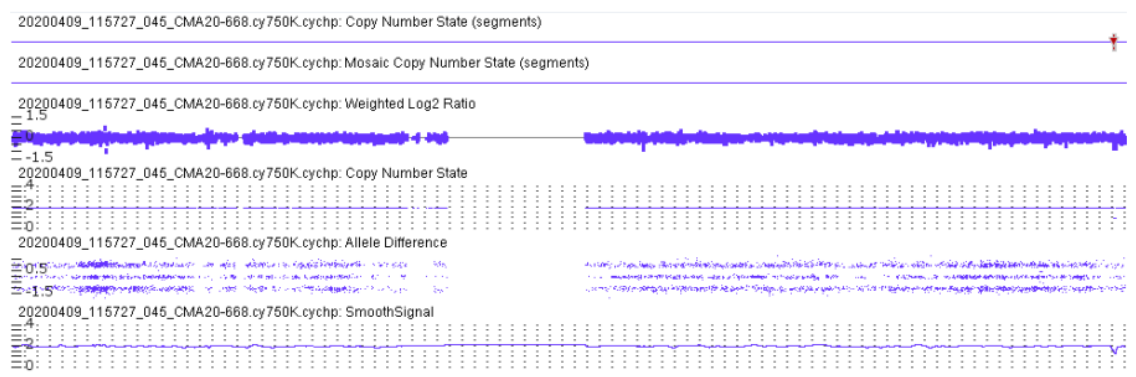

Figure 1 Local magnification of chromosomal aberrations

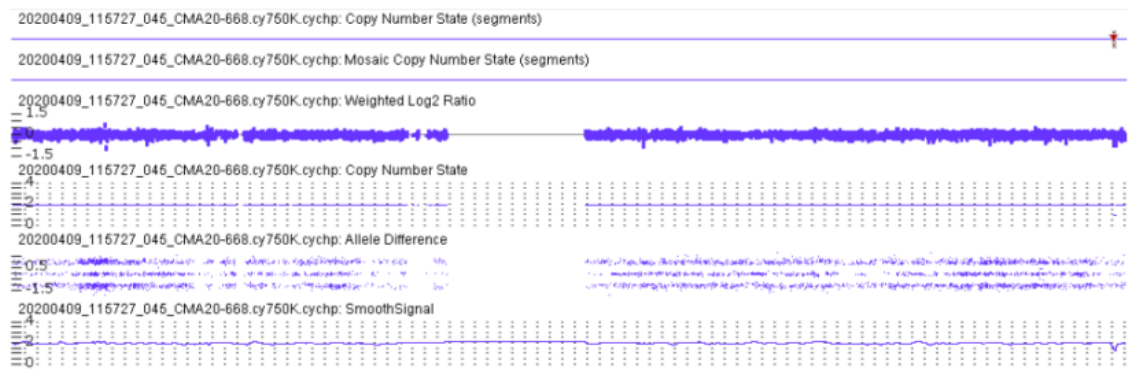

